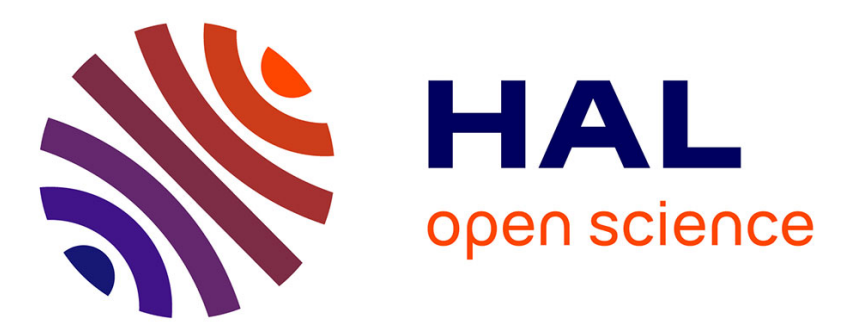

\title{
A re-appraisal of the concept of ideal mixtures through a computer simulation study of the methanol-ethanol mixtures
}

Martina Požar, Bernarda Lovrinčević, Larisa Zoranić, Marijana Mijaković, Franjo Sokolić, Aurélien Perera

\section{To cite this version:}

Martina Požar, Bernarda Lovrinčević, Larisa Zoranić, Marijana Mijaković, Franjo Sokolić, et al.. A reappraisal of the concept of ideal mixtures through a computer simulation study of the methanol-ethanol mixtures. Journal of Chemical Physics, 2016, 145 (6), pp.064509. 10.1063/1.4960435 . hal-01379057

\section{HAL Id: hal-01379057 https://hal.sorbonne-universite.fr/hal-01379057}

Submitted on 11 Oct 2016

HAL is a multi-disciplinary open access archive for the deposit and dissemination of scientific research documents, whether they are published or not. The documents may come from teaching and research institutions in France or abroad, or from public or private research centers.
L'archive ouverte pluridisciplinaire HAL, est destinée au dépôt et à la diffusion de documents scientifiques de niveau recherche, publiés ou non, émanant des établissements d'enseignement et de recherche français ou étrangers, des laboratoires publics ou privés. 


\section{AlP $\mid \begin{aligned} & \text { The Journal of } \\ & \text { Chemical Physics }\end{aligned}$}

\section{A re-appraisal of the concept of ideal mixtures through a computer simulation study of the methanol-ethanol mixtures}

Martina Požar, Bernarda Lovrinčević, Larisa Zoranić, Marijana Mijaković, Franjo Sokolić, and Aurélien Perera

Citation: The Journal of Chemical Physics 145, 064509 (2016); doi: 10.1063/1.4960435

View online: http://dx.doi.org/10.1063/1.4960435

View Table of Contents: http://scitation.aip.org/content/aip/journal/jcp/145/6?ver=pdfcov

Published by the AIP Publishing

\section{Articles you may be interested in}

Hydrophobic hydration and the anomalous partial molar volumes in ethanol-water mixtures

J. Chem. Phys. 142, 064501 (2015); 10.1063/1.4906750

Simulation and reference interaction site model theory of methanol and carbon tetrachloride mixtures

J. Chem. Phys. 132, 084506 (2010); 10.1063/1.3314296

Structural change of ionic association in ionic liquid/water mixtures: A high-pressure infrared spectroscopic study

J. Chem. Phys. 130, 124503 (2009); 10.1063/1.3100099

Molecular-dynamics simulations of dimethylsulfoxide-methanol mixtures

J. Chem. Phys. 123, 154507 (2005); 10.1063/1.2085052

The local structure in liquid methylamine and methylamine-water mixtures

J. Chem. Phys. 113, 8036 (2000); 10.1063/1.1315321

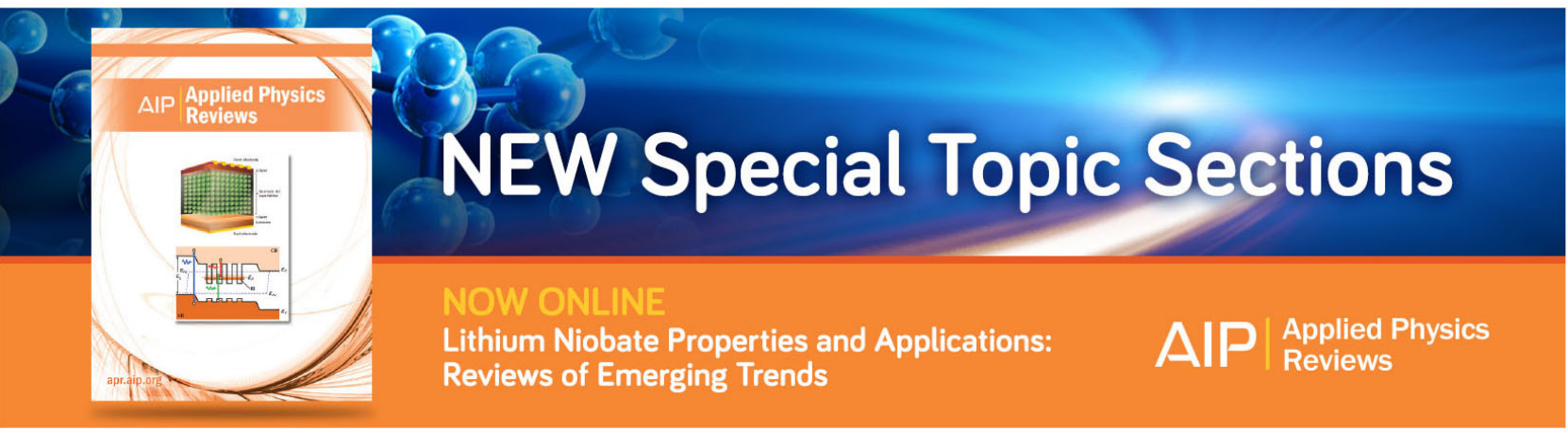




\title{
A re-appraisal of the concept of ideal mixtures through a computer simulation study of the methanol-ethanol mixtures
}

\author{
Martina Požar, ${ }^{1,2}$ Bernarda Lovrinčević, ${ }^{2}$ Larisa Zoranić, ${ }^{2}$ Marijana Mijaković, ${ }^{2}$ \\ Franjo Sokolić, ${ }^{2}$ and Aurélien Perera ${ }^{1, a)}$ \\ ${ }^{1}$ Laboratoire de Physique Théorique de la Matière Condensée (UMR CNRS 7600), Université Pierre et Marie \\ Curie, 4 Place Jussieu, F75252 Paris Cedex 05, France \\ ${ }^{2}$ Department of Physics, Faculty of Sciences, University of Split, Nikole Tesle 12, 21000 Split, Croatia
}

(Received 14 May 2016; accepted 23 July 2016; published online 10 August 2016)

\begin{abstract}
Methanol-ethanol mixtures under ambient conditions of temperature and pressure are studied by computer simulations, with the aim to sort out how the ideality of this type of mixtures differs from that of a textbook example of an ideal mixture. This study reveals two types of ideality, one which is related to simple disorder, such as in benzene-cyclohexane mixtures, and another found in complex disorder mixtures of associated liquids. It underlines the importance of distinguishing between concentration fluctuations, which are shared by both types of systems, and the structural heterogeneity, which characterises the second class of disorder. Methanol-1propanol mixtures are equally studied and show a quasi-ideality with many respect comparable to that of the methanolethanol mixtures, hinting at the existence of a super-ideality in neat mono-ol binary mixtures, driven essentially by the strong hydrogen bonding and underlying hydroxyl group clustering. Published by AIP Publishing. [http://dx.doi.org/10.1063/1.4960435]
\end{abstract}

\section{INTRODUCTION}

The textbook concept of ideal mixtures schematises situations where the different species do not interact with each other or interact similarly. Since molecules always interact through excluded volumes, the concept of ideal mixture concerns mostly enthalpic effects: the excess enthalpy of mixing should be zero. This is never achieved in practice, but many binary mixtures, such as the textbook example benzene-cyclohexane, ${ }^{1,2}$ are nearly ideal. A model example would be a binary mixture of two Lennard-Jonesium (LJ) which are very similar in terms of atomic size and energy parameters, as shown in this study. Since methanol and ethanol are very similar, differing only by a single methyl group, one expects their mixtures would be ideal. The query about the ideality of this type of mixture has been recently asked in a study ${ }^{3}$ of the diffusion constants in mixtures, where the near absence of concentration fluctuations was pointed out. Indeed, the excess energy of the mixture is almost zero, very much like that of the Lennard-Jones mixture tailored over the respective molecular sizes. However, the energies between the two systems are very different, owing to the large electrostatic energies corresponding to the hydrogen bonding. This fact alone raises the question if the alcohol mixtures should be considered as ideal. The answer to this question is not just about the magnitude of the energies, it is more about the local order which results from the highly directional hydrogen bond interaction. This local order manifests itself through the local clustering of molecules, which in turn produces the prepeak in the structure factors. ${ }^{4,5}$ Our principal claim through

\footnotetext{
a) Author to whom correspondence should be addressed. Electronic mail: aup@1ptmc.jussieu.fr
}

this study concerns the existence of two types of disorder in liquids, simple and complex, ${ }^{6}$ and that the signature of the second type should be looked in the site-site structure factors, and eventually in the scattered intensity as measured by X-ray or neutron scattering experiments. This prepeak has been reported for neat alcohols from early experiments. ${ }^{7,8}$ In the case of alcohols, the pre-peak is equally visible in the scattering experiments, ${ }^{9-12}$ as we equally demonstrate through the computer experiments. ${ }^{13}$

Another pathway to speak of ideality in mixtures is through concentration fluctuations. If molecules from different species interact very similarly, their relative distributions will be similar to the respective pure liquid, hence the concentrations fluctuations will be small. The KirkwoodBuff theory allows to relate fluctuations to thermodynamical quantities, such as the chemical potentials. ${ }^{14}$ These latter quantities have an ideal part, related to the counting statistics of the configurations, and an excess part, related to the interactions. The resulting Kirkwood-Buff integrals (KBI), which are the integrals of the pair correlation functions, ${ }^{14,15}$ are a direct test of how interactions affect the mixture, as investigated by various authors. ${ }^{16-23}$ We have shown that some mixtures, such as pentane-benzene, for example, ${ }^{6}$ although having strong excluded volume interactions have near ideal KBI. For this reason, we have termed such mixtures simple disorder. In contrast, most aqueous mixtures are strongly nonideal. ${ }^{15,24}$ However, some aqueous mixtures, such as aqueousdimethylsulfoxyde (DMSO) mixtures are surprisingly ideal from the KBI point of view. ${ }^{25}$ Our interpretation of this ideality is through our finding that, in such mixtures, water forms linear clusters, and the interactions between such clusters and DMSO are near ideal. In other words, although the molecular interaction is very strong, the effective interactions, 
due to clustering, are smaller, and this has a direct observable impact in the thermodynamical properties. The presence of water clusters was witnessed by examination of the snapshots from the simulations, as well as by the presence of a cluster pre-peak in the water oxygen-oxygen structure factor. ${ }^{25}$ This observation is the principal reason for us to examine ideality in alcohol mixtures, where hydrogen bonded clusters are known to exist from scattering experiments.

The concept of ideal mixtures historically concerns first the liquid-gas coexistence through the Raoult Law, and the deviations from it in real mixtures, ${ }^{1,2}$ and its thermodynamical formulation was recently revisited. ${ }^{26}$ The Kirkwood-Buff theory for ideal mixtures has been discussed many times in the literature. ${ }^{27-29}$ Ploetz et al. ${ }^{29}$ have made a detailed comparison of ideal and non-ideal mixtures in comparison with the associated pair correlations. They noted that ideal mixture tends to have very little variation of their correlations in mixing condition with respect to that of their respective pure states, which is what one would expect if all the interactions would be very similar. They equally report the case of benzene-methanol as being strongly non-ideal, in view of the KBIs, but also the fact that the correlation functions of methanol in mixture differ very strongly from those in pure methanol. Our study of aqueous-DMSO ${ }^{25}$ indicates that correlation functions of water in mixing conditions differ widely from that of pure water. However, the KBIs do not reflect this variation and remain quasi-ideal. The reason for this comes from the presence of a cluster pre-peak in the water structure factor, which reduces the fluctuations by absorbing them into the clusters. ${ }^{25}$ This example shows that real space correlations of complex mixtures are tricky to interpret in terms of concentration fluctuations because they show mostly short range features. This example also shows that the concept of ideal mixtures needs to be further clarified in connection to the microscopic distribution of the molecules, which is one of the purposes of this paper. Finally, ideality in condensed matter is an important topic because it allows to evaluate the importance of interactions in various contexts, from ideal quantum statistics of quasi-particles ${ }^{30}$ to the ideal glass concept in the glass transition problem. ${ }^{31}$ From this point of view, the intriguing apparent ideality of complex disorder systems is appealing to investigate in more details.

Through a molecular dynamics study of this alcohol mixture, as well as a well tailored LJ mixture, as an example of simple disorder, we compare the thermodynamic and structural quantities with the aim of sorting out some of the issues raised here. In addition, we study methanol-1propanol mixtures, for which ideality is not expected because of the large difference in the methyl group tails. However, since the hydrogen bonding interaction between the hydroxyl groups is large, one would expect that it could overpower the asymmetry between the methyl tails of the two molecules and lead to a pseudo-ideality. Our study reveals both the reach and the limits of such lines of thinking.

\section{SIMULATIONS}

We have studied binary mixtures of Optimized Potential for Liquid Simulations (OPLS) ${ }^{32}$ and Transferable Potential for Phase Equilibria (TraPPE) $)^{33}$ models of methanol and ethanol, under ambient condition of temperature $\mathrm{T}=300 \mathrm{~K}$ and $1 \mathrm{~atm}$ pressure. The TraPPE model was considered only to test the overall model dependence of the results. Indeed, we do not expect much qualitative difference in terms of structure, although substantial differences are expected for thermo-physical properties, as we have demonstrated in a study of aqueous ethanol mixtures. ${ }^{34}$ We used the TraPPe model for 1propanol. It is noteworthy that the partial charges of the hydroxyl groups are the same between the two models and also for all the hydroxyl groups. In all models and alcohol molecules, the oxygen atom bears a partial charge of $q_{\mathrm{O}}=-0.7 e$ and the hydrogen atom $q_{\mathrm{H}}=0.435 e$. In addition, the methyl group closest to the hydroxyl group has a partial charge of $q_{\mathrm{CH}_{2}}=0.265 e$. The study was conducted by using the GROMACS simulation code. ${ }^{35}$ In parallel, we have tailored two 12-6 Lennard-Jonesium such that the atomic sizes correspond to the experimental molar volumes of methanol and ethanol, respectively. This leads to atomic diameters of $\sigma_{i}=\left(V_{m ; i} \times 10^{6} / 0.6022\right)^{1 / 3}$, where $\mathrm{i}=1,2$ correspond to methanol and ethanol, respectively, with $V_{m ; 1}=40 \mathrm{~cm}^{3} / \mathrm{mol}$ and $V_{m ; 2}=58 \mathrm{~cm}^{3} / \mathrm{mol}$. This gives LJ diameters $\sigma_{1}=3.85 \AA$ and $\sigma_{2}=4.35 \AA$, respectively. We have considered energy parameters $\varepsilon_{1}=1.55 \mathrm{~kJ} / \mathrm{mol}$ and $\varepsilon_{2}=1.6 \mathrm{~kJ} / \mathrm{mol}$, such that the two systems are liquid under ambient conditions. These values may appear too large as compared with usual LJ systems, but the larger diameters should be taken into account as well, which explains that larger energy values are needed. System sizes of $\mathrm{N}=2048$ molecules were used in all cases, which correspond to box sizes of about $55 \AA$. The simulations were performed in the isothermal isobaric (constant NPT) ensemble, with temperature $T=300 \mathrm{~K}$ maintained constant using a modified Berendsen thermostat and pressure maintained constant using the Parrinello-Rahman barostat (both with a time constant of $0.1 \mathrm{ps}$ ). The leap-frog integrator time step was fixed at 1 fs. We followed the same protocol for all of our simulations. All initial configurations were generated using the PACKMOL package ${ }^{36}$ with appropriate pdb (protein database) files for each molecule. The system was then energy minimized, followed by constant NVT simulations of $0.5 \mathrm{~ns}$ performed to obtain an initial equilibrium configuration. A 2 ns run was subsequently performed in the NPT ensemble, which allowed us to reach the 1 atm pressure in all the cases. Production runs were performed for 4 ns runs, for collecting the site-site correlation functions as smooth as possible.

Although classical force fields do not represent the real systems with respect to all aspects of their physical properties, the force fields we have used have been thoroughly tested by the developers ${ }^{32,33}$ for reproducing important thermodynamical properties (the TraPPE force field is tailored to reproduce the phase diagram quite accurately) in the liquid state. This would justify using the Coulomb interactions with partial charges on atomic sites, as well as Lennard-Jones interactions and Lorentz-Berthelot combining rules for the dispersion interactions. We are therefore led to assume that our results must represent the real system mixture properties to a good extent. 
In addition to reporting the site-site correlation functions $g_{a_{i} b_{j}}(r)$, where $i, j \in\{1,2\}$ correspond to the species index and $a_{i}, b_{j}$ represent any two atomic sites on the molecules, we equally report the corresponding structure factors defined as ${ }^{37}$

$S_{a_{i} b_{j}}(k)=\delta_{a_{i} b_{j}}+\rho \sqrt{x_{i} x_{j}} \int d \vec{r}\left[g_{a_{i} b_{j}}(r)-1\right] \exp (i \vec{k} \cdot \vec{r})$,

where $\rho$ is the number density of the system and $x_{i}$ the mole fraction of species $i$. The site-site structure factor reported here is calculated by direct Fourier transform of the site-site correlation functions by standard numerical methods. ${ }^{38}$ When reporting structure factors, for pictorial convenience as in all our previous publications, we replace $\delta_{a_{i} b_{j}}$ by 1 .

In order to appreciate the amount of concentration fluctuations in these mixtures, we have computed the Kirkwood-Buff integrals (KBI) from the structure functions obtained from the simulations,

$$
G_{i j}=\int d \vec{r}\left[g_{a_{i} b_{j}}(r)-1\right] .
$$

In order to obtain the correct version of these KBI, we have adjusted the asymptotes of the correlation functions $g_{a_{i} b_{j}}(r)$ for the Lebowitz-Percus correction, ${ }^{39}$ following procedures that we have outlined in previous works. ${ }^{40,41}$ In order to test the proper asymptotes, we always consider the running KBI (RKBI) defined as ${ }^{14}$

$$
G_{a_{i} b_{j}}(r)=4 \pi \int_{0}^{r} d s s^{2}\left[g_{a_{i} b_{j}}(s)-1\right],
$$

whose asymptote should be perfectly horizontal and tend towards the correct KBI value $G_{i j}$. Fig. 1 shows a

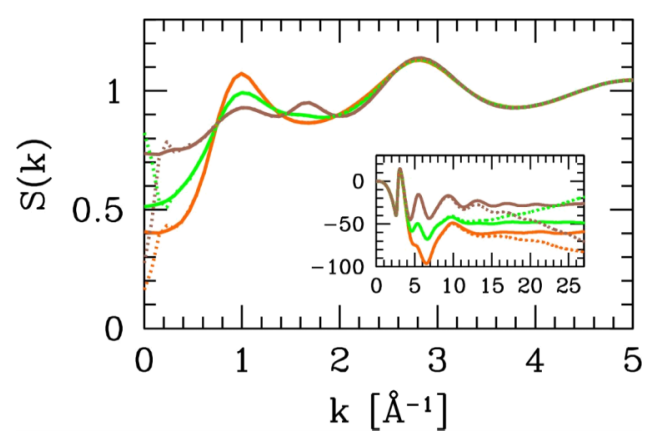

FIG. 1. Structure factors and RKBI (inset) for the equimolar methanolethanol mixture, demonstrating the Lebowitz-Percus asymptote problem (see text). Methanol correlation is show in brown, ethanol in orange, and cross correlations in green.

demonstration of this problem for the equimolar mixture. The uncorrected RKBI are shown by dashed lines in the inset, while the corrected ones are in full lines. The corresponding structure factors are shown in the main panel. One sees very clearly the dramatic effects (tail of the $g_{a_{i} b_{j}}(r)$ or small-k behaviour of $S_{a_{i} b_{j}}(k)$ ) that appear from the use of uncorrected data taken directly from the simulations.

\section{RESULTS}

\section{A. Microscopic structure analysis}

\section{Snapshots}

In Fig. 2, we show typical snapshots for both types of systems for $x_{E}=0.2,0.5$, and 0.8 , where $x_{E}$ represents the
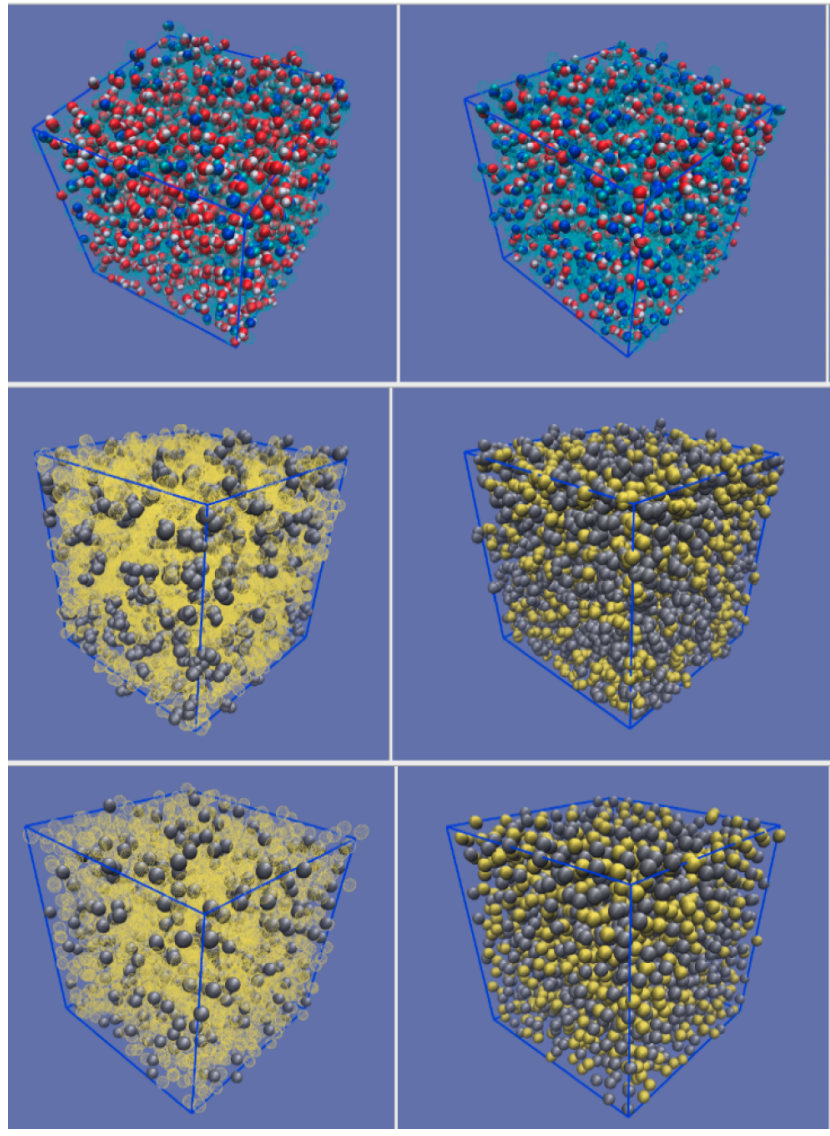
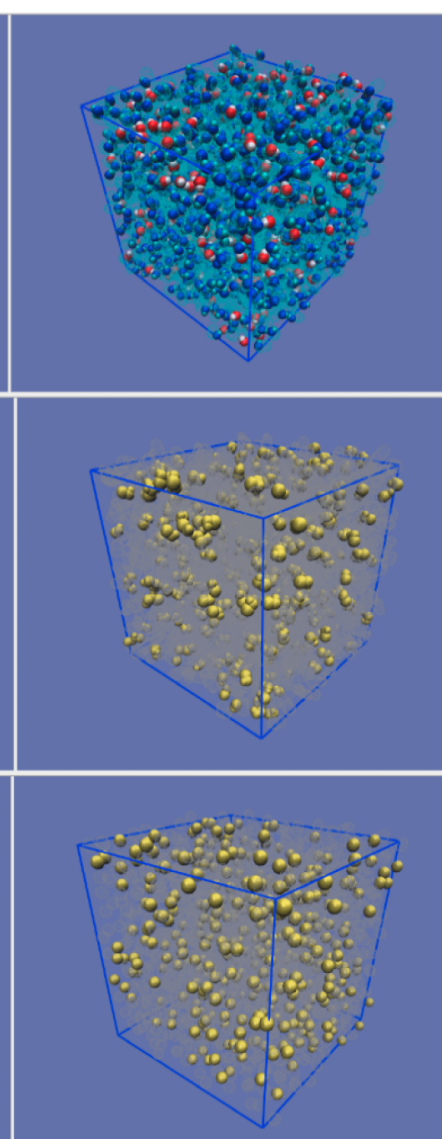

FIG. 2. Snapshots. (Top and middle) Methanol-ethanol mixtures for $x_{E}$ $=0.2,0.5$, and 0.8. Top panel shows only the bonding sites (methanol oxygens in red and ethanol oxygens in cyan, all hydrogens in white), and the middle panel shows the full molecules (methanol in yellow and ethanol in grey); (bottom) LJ mixtures for the same concentrations and same color conventions as the middle panel. 
mole fraction of the component 2 (ethanol for the alcohol mixtures). The top panel snapshots show the bonding sites (oxygen and hydrogen, with the oxygen shown in red for methanol and cyan for ethanol) to illustrate the fact that both species hydroxyl groups mingle into a network structure that covers the entire box. This is most visible when rotating the box when only the hydroxyl groups are shown. A close examination of the shown snapshot shows strings of hydrogen bonded hydroxyl groups. We observe no micro-segregation of the two types of groups. This is due to the fact that the partial charges on the oxygen and hydrogen sites are exactly the same for both species in both models. The middle and lower panel illustrate the global heterogeneity (ethanol in grey and methanol in yellow, with similar conventions for the LJ system shown in the bottom panel). The global observation is that the hydrogen bonding does not bring out any specific form of local heterogeneity. In other words, both the alcohol and the LJ mixture look very similar. This enforces the impression of ideality of both systems.

\section{Correlations}

The correlation functions are shown in Fig. 3 for the same three typical concentrations. One sees that there is a huge difference between the correlations $g_{\mathrm{O}_{a} \mathrm{O}_{b}}(r)$ of the various oxygen atoms $\mathrm{O}_{x}$. in the alcohol mixtures (left panels) and those in the Lennard-Jonesium mixtures (dashed lines in the right panels). In contrast, the correlations between the methyl groups (right panel) look very much like those between the Lennard-Jonesium, as shown in the insets. This fact alone demonstrates the strong local order that can be created

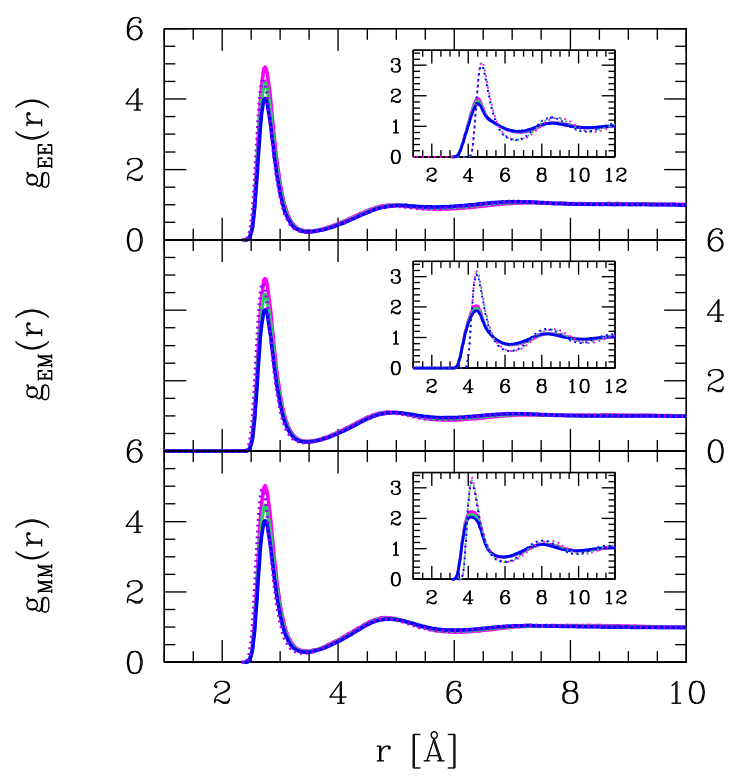

FIG. 3. Selected site-site correlation functions. The various oxygen-oxygen correlations are shown in the main panels, and the methyl-methyl correlations as well as the LJ correlations (dashes) are equally represented in the insets. The two top panels show the ethanol-ethanol oxygen correlations, the two middle show the ethanol-methanol oxygen correlations, and the two lower the methanol-methanol oxygen correlations. Each data set is shown for 3 solute concentrations $x_{E}=0.2$ (blue), 0.5 (green), and 0.8 (magenta). The TraPPE model correlations are shown only for $x_{E}=0.5$ in dotted purple lines (only on main panels). from the hydrogen bonding between the hydroxyl groups. Indeed, it is well known that H-bond induced clustering in neat alcohol leads to well defined cluster shapes, such as chains and loops. ${ }^{13,12}$ Interestingly, we note that all the OO correlations look very much alike and independent of the concentration. This can be partly explained by the fact-as noted in Subsec. III A 1 - that the partial charges on the oxygen and hydrogen atoms are the same for both alcohols. But it also indicates that the correlations between these two molecules are also very similar. This feature enforces the interpretation of perfect mixing between the two alcohols. To close this sub-section, we note that the oxygen-oxygen correlations for the TraPPE model, shown in the main panels in dashes, are indistinguishable from the OPLS data.

\section{Structure factors}

Fig. 4 shows the structure factors corresponding to the correlation functions shown in Fig. 3. We immediately note, in the left panel the oxygen-oxygen pre-peak in the $S_{\mathrm{O}_{a} \mathrm{O}_{b}}(k)$ around $k_{P P} \approx 0.8 \AA^{-1}$, which does not appear in the other sitesite functions (right panels), and whose main peak is centered about the size of the methyl site $\left(k_{M P}=2 \pi / \sigma_{M} \approx 1.7 \AA^{-1}\right.$, for $\sigma_{M} \approx 3.4 \AA$ ). These pre-peaks witness the cluster-cluster interactions, much the same way the main peak witness the corresponding atom-atom interactions. It is more marked for the ethanol-ethanol function, indicating the preferential hydrogen bonding between the species. It should be noted that the pre-peak does not correspond to the strong main peak in the oxygen-oxygen correlations but to the longer range correlations between these atoms. Indeed, the distance between the oxygens is about $r \approx 2 \AA$ which is smaller than the methyl-methyl contact $\sigma_{\mathrm{M}_{a} \mathrm{M}_{b}} \approx 3.3 \AA$, and consequently the corresponding wavelength should be at the larger $k \approx 2 \pi / \sigma$, which is clearly not the case. Therefore, the pre-peak should

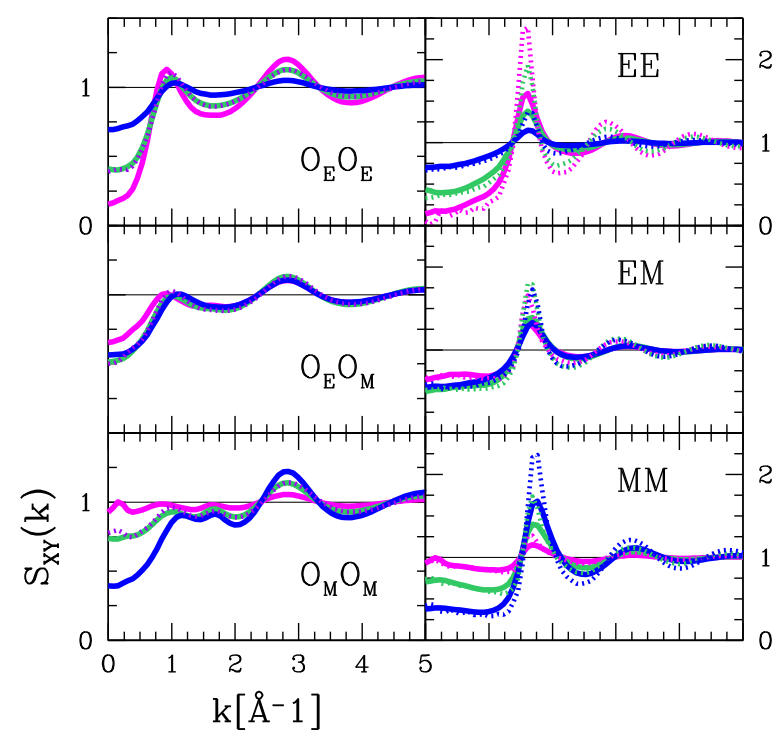

FIG. 4. Selected site-site structure factors for the correlations shown in Fig. 3, with same line and color conventions. The left panels represent the structure factors for the various oxygen-oxygen correlations, while the right panels show the structure factors corresponding to the insets in Fig. 3. 
necessarily correspond to interactions/correlations between larger objects, namely, the clusters of hydrogen bond network. The existence of such object witnesses an inherent complexity which is absent from the simple Lennard-Jonesium mixtures. The fact that this complexity is hidden behind several apparent signatures of simplicity is worth examining, which we will do in Sec. IV. Once again, the TraPPE model structure factors are shown as dashed lines only for the oxygen correlations in the left panels and are seen to be not so different from the OPLS data.

\section{Cluster analysis}

The aggregate structures can be conveniently studied in computer simulations, by specifying a particle connectivity criteria to include two particles inside a cluster, and then simply collect statistically all such clusters. Here, we use the used Stillinger distance criteria ${ }^{42}$ where the cutoff distance is defined by the first minima of the particle-particle radial distribution function. This way, the interactions between bonded particles are indirectly related to their interactions through the radial distribution function. The cluster size distributions are calculated for the clustering of the like-like sites, using several different statistical approaches. We show the results for the cluster size probability functions,

$$
s_{n}=\frac{\sum_{k=1}^{N_{c}} s(n, k)}{\sum_{k=1}^{N_{c}} \sum_{j=1}^{N_{m o l}} s(j, k)},
$$

where $s_{n}$ is the probability for the cluster formed of $n$ sites, $s(k, n)$ represents the number of clusters of the size $n$ in the configuration $k$. The cutoff distances defined in this work are $r_{c}=3.5 \AA$ between the oxygens and $r_{c}=4.5 \AA$ between the methyl groups. The cluster distributions between different pairs of sites are displayed in Fig. 5. The main panel shows the distributions for the oxygen atoms (methanol in dotted-dashed lines and ethanol in full lines), while the inset is for the carbon atoms (with same conventions). Pure methanol is shown in dotted-dashed grey line and pure ethanol in dotted black line. We note that each of

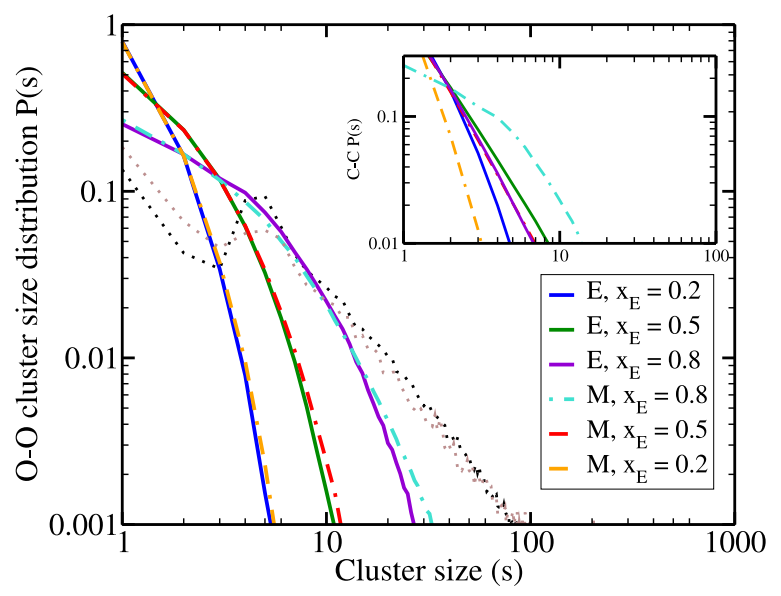

FIG. 5. Cluster distribution of the oxygen atoms (main panel) and carbon atoms (inset), for different ethanol mole fractions. Dot-dashed lines for methanol (labelled M) and full lines for ethanol (labelled E). Pure ethanol and methanol are shown in dotted lines, in black and grey, respectively. these curves are strongly non-monotonous have a specific peak at about cluster size 5, which is consistent with the hydroxyl group cluster chains and loops, which we visualise in the corresponding snapshots. This specific peak disappears with addition of cosolvent, and the distributions become fully monotonous. In all cases, we note that the lower the concentration of a species, the smaller its cluster distribution. This is intuitively obvious since clusters should be smaller with smaller number of particles. Since the specific peak corresponds to a specific cluster geometrical shape-namely, chains - their disappearance in mixing condition does not mean that these chain structures have disappeared but that they are now chains made of mixed methanol-ethanol hydroxyl groups. So this disappearance of the specific is a direct indication of the perfect mixing of methanol and ethanol. It confirms the analysis reported above from other structural analysis. The inset shows trivial distributions of the carbon atoms, which witness random distribution of these atoms.

\section{B. Thermodynamical analysis}

\section{Volumes and energies}

In Fig. 6, we report configurational energies (top panel), volumes (bottom panel), as well as associated excess quantities (as insets), for both mixture models, as a function of ethanol mole fraction. Experimental volumes ${ }^{43}$ are equally reported. It is seen that both the OPLS models for neat methanol and ethanol are not very accurate, and in different ways for each models. This is a typical issue with model force fields of realistic systems. As a consequence, the excess volumes are very different in magnitude. However, we observe that the OPLS mixtures show a positive excess volume, when typical hard sphere mixtures show negative excess volume. ${ }^{1}$ Indeed, the LJ mixture shows exactly that, as well as the TraPPE model

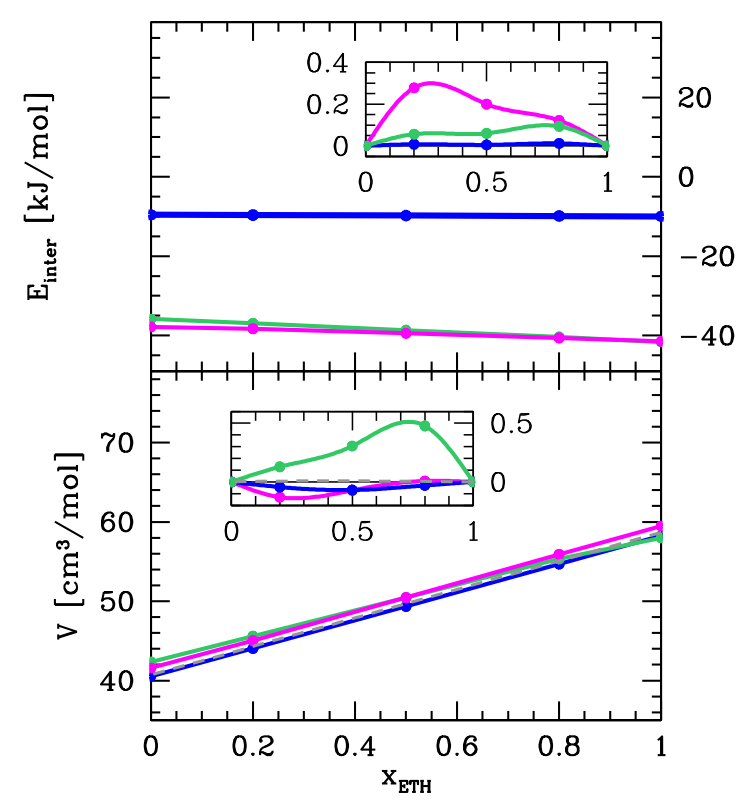

FIG. 6. Enthalpies (top panel), molar volumes (lower panel), and corresponding excess quantities (in the insets). Green for the OPLS model, magenta for the TraPPE model, and blue for the LJ model. The experimental data for the volumes from Ref. 43 are equally shown in dashed grey lines. 
in the small ethanol mole fraction region. Therefore, even for the experimental system, there is an unfavourable enthalpic contribution, which compensates the typical excluded volume effects. The OPLS mixture overestimates this compensation. Several issues concerning the force fields could produce the type of discrepancy, such as for example the neglect of polarisability. One can search after models that can reproduce better experimental data. We have examined in a previous study $^{34}$ several models of ethanol and found that none of them reproduced accurately the experimental enthalpies and volumes of aqueous-ethanol mixtures. With respect to this type of "fitting problem," we adopt the attitude that these small inaccuracies do not alter the global microscopic structure of the mixture, which remains a good representative of the real system in all cases.

Perhaps the most important feature in the top panel of Fig. 6 is the fact that the alcohol mixtures have negative configurational energies (about $-40 \mathrm{~kJ} / \mathrm{mol}$ ) far below that of the LJ mixtures (about $-10 \mathrm{~kJ} / \mathrm{mol}$ ). This type of energy cannot be obtained by altering the $\varepsilon$ energy parameters on the LJ system, since increasing the interaction well depth will likely lead to crystallisation. These large negative energies are therefore a signature of an hidden complexity. We will come back to this point in Sec. IV.

There is very little energy change between the two neat alcohols, as well as their LJ representatives. The excess energies are positive for all types of mixtures, indicating that the mixing is slightly less favourable than the neat states. It is interesting that the OPLS model overestimates the excess in the ethanol rich side, while the TraPPE model does that in the methanol rich side. These small glitches demonstrate the low reliability of force fields in general as far as thermo-physical properties are concerned.

\section{Kirkwood-Buff integrals}

In Fig. 7, we report the KBI for both mixture models (symbols), together with the expected ideal KBI (lines). These ideal $\mathrm{KBI}$ are defined from the relations derived from the KB

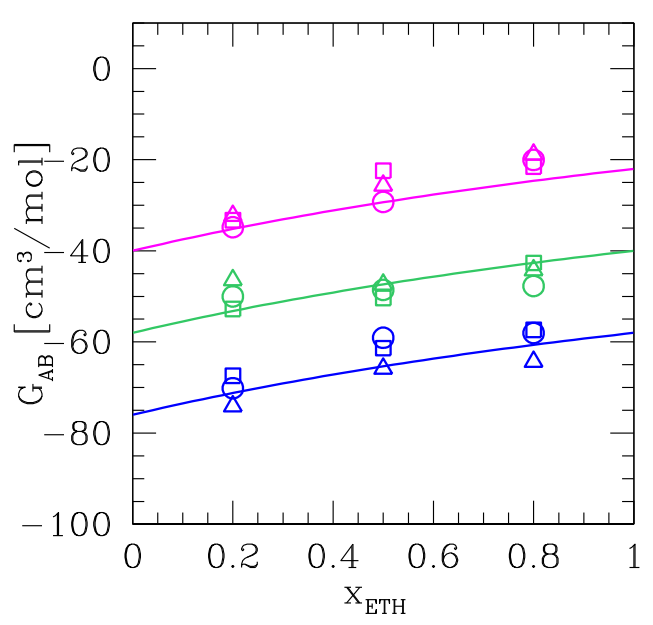

FIG. 7. Kirkwood-Buff integrals for the alcohol (OPLS in open dots, TraPPE in open squares), LJ (open triangles and ideal KBI in lines) mixtures. The solvent-solvent (methanol) KBI are in magenta, the solute-solute (ethanol) in blue, and the cross in green. theory, ${ }^{14,15,24}$

$$
G_{i j}=\left[k_{B} T \kappa_{T}-\frac{\bar{V}_{i} \bar{V}_{j}}{V D}\right]\left(1-\delta_{i j}\right)+\frac{1}{x_{i}}\left[\frac{\bar{V}_{j}}{D}-D\right] \delta_{i j},
$$

where $\kappa_{T}$ is the isothermal compressibility $\left(k_{B}\right.$ is Boltzmann constant and $T$ the temperature), $\bar{V}_{i}$ is the partial molar volume of species $i, V$ the total volume, and $D$ is related to the concentration fluctuations through the expression, ${ }^{14,15,24}$

$$
D=x_{i}\left(\frac{\partial\left(\mu_{i} / k_{B} T\right)}{\partial x_{i}}\right)_{T P},
$$

where $\mu_{i}$ is the chemical potential of species $i$. The ideal KBI correspond to neglecting concentration fluctuations by setting $D=1$, which amounts to consider ideal chemical potentials $\mu_{i}=k_{B} T \ln \rho_{i}$ (where $\rho_{i}=\rho x_{i}$, with $\rho=N / V$ the total density, and $x_{i}$ the mole fraction of species $i$ ) - in other words, non-interacting species. It is quite apparent that both set of data are quite similar, although the alcohol mixtures show weak non-ideality. These results alone strongly suggest that these mixtures are ideal from thermodynamical point of view, with very little concentration fluctuations. The near absence of such fluctuations shows a uniform distribution of the molecular species, which is what we have observed in the snapshots and the correlation function analysis. This homogeneity is true, despite the underlying strong hydrogen bonding and clustering.

\section{Scattering analysis}

One way to investigate the structure of liquids is through $\mathrm{X}$-ray or neutron scattering. The scattered intensity in each case can be in fact obtained through the knowledge of atomatom structure factors $S_{a_{i} b_{j}}(k)$ in Eq. (1), from the canonical expression, ${ }^{44}$

$$
I(k)=\rho \sum_{i, j} x_{i} x_{j} \sum_{a_{i} b_{j}} f_{a_{i}} f_{b_{j}} \tilde{h}_{a_{j} b_{j}}(k),
$$

where $x_{i}$ is the mole fraction of species $i, a_{i}$ is the index of the atom of type $a$ in molecule of species $i, f_{a_{i}}$ is the atomic form factor associated to atom $a_{i}$, and the function $\tilde{h}_{a_{i} b_{j}}(k)$ is the Fourier transform of $h_{a_{i} b_{j}}(r)=g_{a_{i} b_{j}}(r)-1$. From Eq. (1), we obtain the expression relating to the site-site structure factors,

$$
I(k)=\sum_{i, j} \sqrt{x_{i} x_{j}} \sum_{a_{i} b_{j}} f_{a_{i}} f_{b_{j}}\left(S_{a_{j} b_{j}}(k)-\delta_{a_{i} b_{j}}\right) .
$$

Interestingly, for $k=0$, as can be seen from Eqs. (2) and (3), $I(k=0)$ is related to the $\mathrm{KBI} G_{i j}$ through the following form:

$$
I(k=0)=\sum_{i j}\left(\sum_{a_{i} b_{j}} f_{a_{i}} f_{b_{j}}\right) G_{i j},
$$

which displays a highly non-trivial dependence on the weighting atomic form factors. In particular, the concentration dependence of the $I(k=0)$ cannot be simply inferred from that of various KBI.

The atomic form factors $f_{a_{i}}$ depend on $k$ for X-ray scattering and are independent of $k$ for neutron scattering. ${ }^{44}$ This is due to the underlying influence of electrons versus nucleons. These functions are found in tables. ${ }^{45}$ The structure factors are provided by the simulations, with a slight difference 


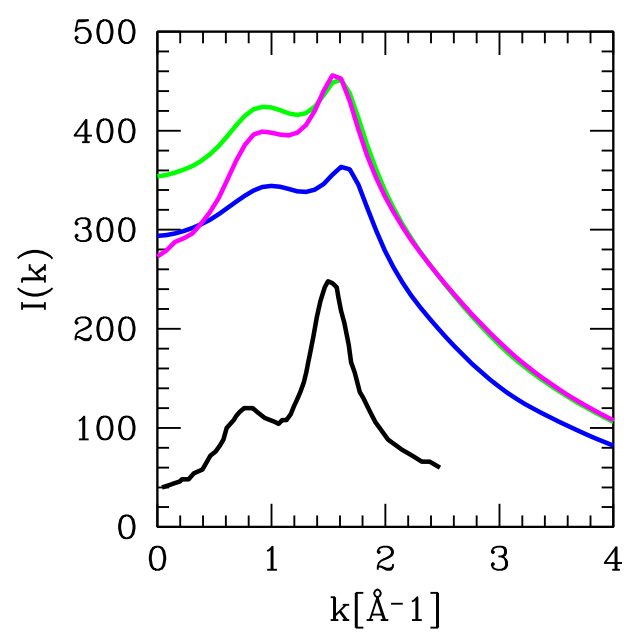

FIG. 8. X-ray scattering intensities $I(k)$, as obtained from the simulations of the methanol-ethanol mixtures through Eq. (3). The blue is for ethanol mole fraction $x=0.2$, green for $x_{E}=0.5$, and magenta for $x_{E}=0.8$. The curve in black is neat ethanol experimental data from Ref. 48 , scaled to compare with simulations (see text).

however. The OPLS force field models the $\mathrm{CH}_{n}$ methyl groups as a single united atom. While it is possible to artificially recover the individual $\mathrm{C}$ and $\mathrm{H}$ atom contributions by using the "free-rotation" approximation, ${ }^{46,47}$ we have made the approximation of considering the carbon atom form factor for the united atoms $\mathrm{CH}_{n}$. This approximation is justified only from how close the resulting $I(k)$ matches the experimental one.

Fig. 8 shows the $\mathrm{I}(\mathrm{k})$ for the three typical ethanol mole fractions. In addition, the experimental data for pure ethanol ${ }^{48}$ is shown in black (the magnitude of the experimental data is given in arbitrary units, as usual, so we scaled it with an appropriate factor such that it matches the scale of the data reported here). We note that the main peaks and pre-peaks appear distinctly in both sets of data and positioned at nearly the same k-vector positions. The small mismatch could arise from atomic size discrepancy inherent to the OPLS force fields. The important fact is the presence of a pre-peak in both the experimental and simulated data. In particular, from Eq. (5), one sees that one cannot infer the underlying complexity due to the pre-peak, solely from the $G_{i j}$ obtained at $k=0$, which reflects no concentration fluctuations in the present case.

\section{The methanol-1propanol mixtures}

This mixture is studied in order to examine how far the dominance of the hydroxyl group interaction would dominate the fate of the mixture, if longer alcohol chains were used. As said in the Introduction, it is a competition between the strong asymmetry of the alkyl tails of methanol and 1 propanol, and the strong and identical interaction between the hydroxyl groups. Fig. 9 resumes the essential findings for the equimolar mixture of methanol and 1propanol. Other concentrations show results which are overall very similar. Fig. 9(a) shows $g_{\mathrm{OO}}(r)$ between the oxygen atoms of methanol in methanol-1propanol(blue curve) and methanolethanol mixtures(magenta curve). The inset shows the $g_{\mathrm{OO}}(r)$ for the oxygens of 1propanol (green curve) and ethanol (orange curve). Fig. 9(b) shows the structure factors $S_{\mathrm{OO}}(k)$ corresponding to the $g_{\mathrm{OO}}(r)$ of the panel (a). We observe that the first peaks in Fig. 9(a) are not so much different than those shown in Fig. 3. The second peak shows some differences, which are expected because of the difference between the molecules. The structure factors show that the pre-peak is nearly identical between all these systems, and the only changes are in long range correlations, i.e.,very small $\mathrm{k}$-values, which is also expected. These two plots confirm that the hydroxyl group correlations are the same for both mixtures. Fig. 9(d) shows a snapshot, with the hydroxyl groups of methanol shown in red(oxygen) and white(hydrogen), while
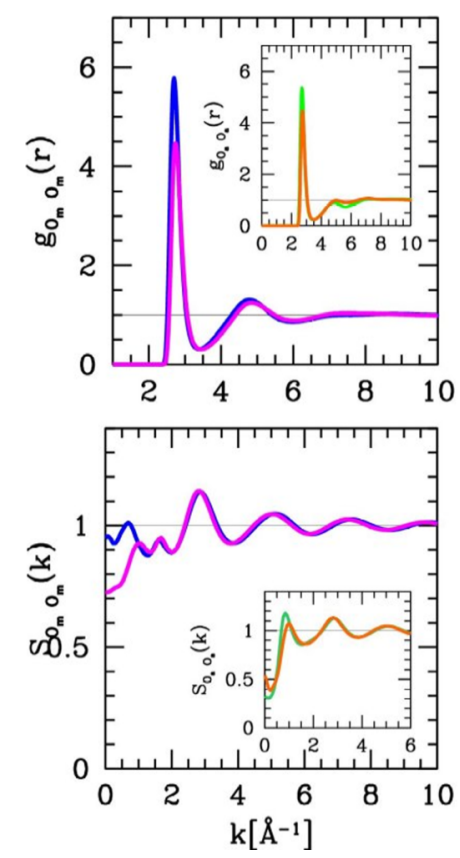

(a)

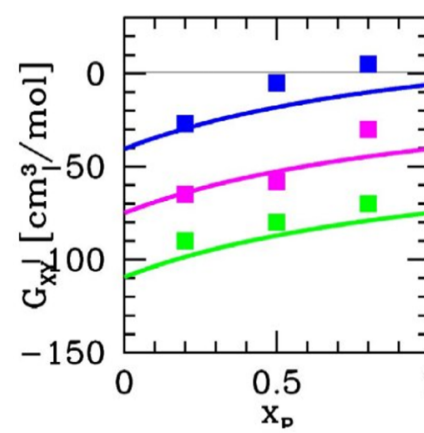

(b)

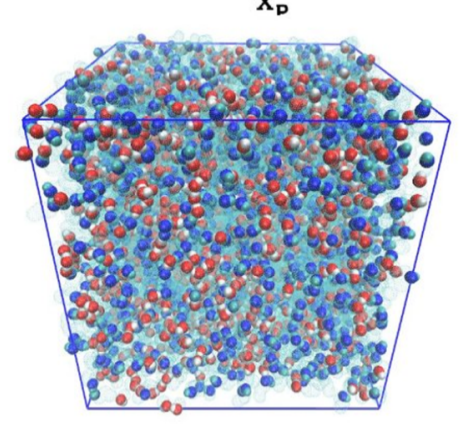

(c)

(d)
FIG. 9. Data for the equimolar methanol-1propanol mixture. (a) Plot of the methanol oxygen-oxygen correlation functions $g_{\mathrm{OO}}(r)$ (blue) compared with the same in methanol-ethanol equimolar mixture (magenta). The inset shows $g_{\mathrm{OO}}(r)$ for 1propanol oxygens (green) compared with that between the ethanol oxygens in equimolar methanol-ethanol mixture (orange). (b) The structure factors corresponding to $g_{\mathrm{OO}}(r)$ data in (a). (c) The KBI for the methanol-1propanol mixture: $G_{\mathrm{M}-\mathrm{M}}$ in blue, $G_{1 \mathrm{P}-1 \mathrm{P}}$ in green, and cross $G_{\mathrm{M}-1 \mathrm{P}}$ in magenta (lines for the ideal curves and symbols for simulations). (d) Snapshot of the equimolar methanol-1propanol mixture. Methanol oxygens shown in red, hydrogens in white, 1propanol oxygens in blue, and hydrogens in cyan. All methyl united atom groups show as semi-transparent cyan spheres. 
those of 1propanol are shown in blue (oxygen) and cyan (hydrogen). The methyl groups are indifferently shown as cyan semi-transparent spheres. An immediate feature is the existence of the chain-like aggregates of the hydroxyl groups, with random distribution of the methanol and 1propanol hydroxyl groups. The rotation of the snapshot clearly shows the network structure, very similar to that observed in the case of Fig. 2. Fig. 9(c) shows the KBI from the simulation (symbols) compared with the ideal KBI plotted in lines. The agreement is quite good, hinting that the fluctuations are near ideal, like in the methanol-ethanol system. However, we note that there are small but systematic deviations from the pure ideality. This study indicates that it is very likely that mono-ol mixtures, specifically of closely related molecules, are very likely to be quasi-ideal, with the ideality being a result of the identity of the dominating hydroxyl group energies. It would be interesting to explore the limits of such ideality, in particular when alcohols become branched or when the number of hydroxyl groups is increased.

\section{DISCUSSION}

The various quantities reported in Subsec. III B above show the similarity between the two types of systems-simple and complex disorder, as far as the ideality is concerned, despite the large differences in energy between the two systems-as shown in Fig. 6. Perhaps one signature that could raise some suspicion about the ideality is the difference in magnitude of the energies. For example, one could imagine making the LJ $\varepsilon$ energy parameter of the second mixture large enough to match the energies of the alcohol mixtures. However, this amounts to decrease the temperature of the mixture, and doing so will lead to a solid phase. In other words, it is impossible to change the $\varepsilon$ in order to match both the liquid state and the energy of the alcohol mixture. One explanation is that it is impossible to match the magnitude of the Coulomb energy with the LJ form. But this explanation hides the true origin of the difference. In fact, it is the directionality of the interaction which is responsible for this difference. More fundamentally, it is the asymmetry of the interaction in the alcohol system versus the symmetric interaction in the $\mathrm{LJ}$ system, which is responsible for the complexity of the first system. Nature has used the Coulomb interaction through the electronic structure of the atoms, but one can build a model with purely LJ-type interactions, asymmetrically disposed in the molecules, which would create the same complexity. In such model, the energies would probably be closer to those of the simple LJ mixture, but the tendency to form bonded cluster would equally exist and will appear through a pre-peak in the structure factors. A simple such model has been produced recently in our group, ${ }^{49}$ which is able to show the clustering behaviour of complex mixtures.

This type of exercise allows one to better understand the origin of complexity in disordered matter. In Section III B 1, we observed that the alcohol mixtures have a much more negative configurational energy than the LJ mixtures. Since they nevertheless look ideal from several aspect, one can ask how their complexity is hidden behind apparent ideality. This question should be brought up under the light of the enthalpy-entropy compensation (EEC) principle ${ }^{50-53}$ which is particularly important in biophysics. ${ }^{50,54}$ This principle states that two different systems 1 and 2 can have a deceitfully similar free-energy $G_{1}=G_{2}$, with $G_{i}=H_{i}-T S_{i}$, where $H_{i}$ is the enthalpy and $S_{i}$ the entropy of system $i$, while one has $H_{2}=H_{1}+\delta H$ and $S_{2}=S_{1}+\delta S$, such that $\delta H=T \delta S$. This leads to two different cases of EEC, depending on the sign of $\delta H$. The most natural way is $\delta H<0$, which is also the most commonly addressed..$^{50}$ In this case, the enthalpy $H_{2}$ of the second system is more negative, while its entropy $S_{2}$ is smaller than that of the first. In other words, the second system is more ordered and this order is the result of a larger attraction between particles. This principle does not necessarily imply that such particles would form clusters, but it is obvious that a cluster structure would favour more order and less entropy. On the other hand, it is quite clear that attractive directional interactions necessarily imply both particle association and clustering. This is what we observe in the case of the methanol-ethanol mixtures. The second EEC mechanism is when $\delta H>0$, which is less discussed. For example, it could correspond to the so-called core-soft systems, ${ }^{55-57}$ where increased repulsion equally results in the appearance of local clustering. ${ }^{58,59}$ However, this type of order necessitates that the system is constrained by volume, ${ }^{55,56}$ hence it will have very large pressures and the $V \delta P$ term appears in the free-energy. This is the required condition for the re-ordering of the system, and the reduction of the entropy is compensated by the increase in pressure. Therefore, the EEC principle in this second type of system is rather artificial.

The type of ideality that we discuss herein is related to complex mixtures, and we have shown how this ideality differs from the textbook concept, which concerns principally simple disorder mixtures. In order to emphasize this type of ideality, which hides very strong local molecular organisations, we wish to propose the term of "super-ideality," analogy with similar naming in condensed matter physics, as mentioned in the Introduction.

Finally, we would like to connect this work with our earlier concerns on the universality of micro-heterogeneity in mixtures with complex disorder. ${ }^{4,5}$ In previous works, we have underlined the importance of micro-heterogeneity in mixtures involving at least one associating liquid. ${ }^{6,41,60-62,25}$ In particular, we have emphasized that this micro-segregation should be witnessed through the presence of a pre-peak in the partial structure factors. In the mixture studied herein, however, we see that there are no such micro-heterogeneity and micro-segregation of each species. On the contrary, the two species seem to mix rather well; and yet, a scattering pre-peak is observed. Despite the apparent contradiction, we wish to underline that the presence of the pre-peak in the partial structure factors is solely due to the presence of specific clusters, whether they arise from self-segregation of one species with respect to the others, or clustering of hydroxyl groups within mixing - such as that observed here. In both cases, the clusters provide a supplementary modulation of the correlation functions, in addition to the site-site modulation. The present work should help clarify this important issue. 


\section{CONCLUSION}

The fact that mixtures of isomers of alcohols (e.g., 1-butanol and 2-butanol) are ideal is well known from textbooks. However, the usual analysis is in terms of energy and free-energy differences. However, through the present analysis, we have underlined that the macroscopic analysis could mask the underlying complexity, characterized in particular by the presence of intense clustering interactions, witnessed by a pre-peak in the structure factor and the scattering intensity. This analysis shows that conclusions made on the sole $I(q=0)$ or $S_{i j}(q=0)$, which represent the macroscopic limit, ${ }^{37}$ cannot reveal the underlying complexity, namely, the pre-peak structure. This pre-peak structure represents the cluster-cluster interactions/correlations, while the usual main peak represents the atom-atoms correlations/interactions. In some cases, this underlying complexity can be hidden, which is at the origin of the entropy-enthalpy compensation principle.

This work leaves several issues open for future investigations. One of them is why aqueous mixtures of simple alcohol molecules have scattering functions without pre-peak, when computer simulations show the clear evidence of domain segregation, which leads naturally to the existence of such pre-peaks. In other words, there is a cancellation of pre-peaks, which occurs for some systems and not for others. For example, this scattering pre-peak is present for alcohol mixtures, room-temperature ionic liquids, ${ }^{63,64}$ mixtures of alcohols and ionic liquids, ${ }^{65}$ some aqueous mixtures with diols and triols. ${ }^{66,67}$ They are absent for many aqueous mixtures ${ }^{66}$ and for now there is no method to predict which mixtures show such a pre-peak or not. This important problem has not raised the attention of the community because the omni-presence of pre-peaks in atom-atom structure factors has only been recently established, principally through the works in our group. ${ }^{6}$ This is implicitly related to the acknowledgement of the two categories of disorder in disordered systems. ${ }^{6}$ Other issues are related to the microscopic mechanisms for self-assembly and the influence of the complexity of the solute molecule. ${ }^{25}$ These issues should lead to a more precise and hintful description of bio-matter in general. ${ }^{68,69}$

\section{ACKNOWLEDGMENTS}

This work has been partially supported by the Croatian Science Foundation under the Project No. 4514 "Multi-scale description of meso-scale domain formation and destruction."

${ }^{1}$ J. S. Rowlinson and F. L. Swinton, Liquids and Liquids Mixtures (Butterworths Scientific, London, 1982).

${ }^{2}$ P. Atkins and J. de Paula, Atkin's Physical Chemistry (Oxford University Press, New York, 2010).

${ }^{3}$ S. Parez, G. Guevara-Carrion, H. Hasse, and J. Vrabec, Phys. Chem. Chem. Phys. 15, 3985-4001 (2013).

${ }^{4}$ A. Perera, B. Kežić, F. Sokolić, and L. Zoranić, in Molecular Dynamics, edited by L. Wang (InTech, Rijeka, 2012), Vol. 2.

${ }^{5}$ A. Perera, in Fluctuation Theory of Solutions: Applications in Chemistry, Chemical Engineering, and Biophysics, edited by P. E. Smith, J. P. O'Connell, and E. Matteoli (CRC Press Taylor and Francis, 2012), ISBN: 9781439899229.

${ }^{6}$ M. Požar, J. B. Seguier, J. Guerche, R. Mazighi, L. Zoranić, M. Mijaković, B. Kežić-Lovrinčević, F. Sokolić, and A. Perera, Phys. Chem. Chem. Phys. 17, 9885 (2015).

${ }^{7}$ B. E. Warren, Phys. Rev. 44, 969 (1933).
${ }^{8}$ S. Sarkar and R. N. Joarder, J. Chem. Phys. 99, 2032 (1993).

${ }^{9}$ D. G. Montague, J. C. Dore, and S. Cummings, Mol. Phys. 53, 1049 (1984).

${ }^{10}$ T. Yamaguchi, K. Hidaka, and A. K. Soper, Mol. Phys. 96, 1159 (1999).

${ }^{11}$ T. Weitkamp, J. Neuefeind, H. E. Fisher, and M. D. Zeidler, Mol. Phys. 98, 125 (2000).

${ }^{12}$ J.-H. Guo, Y. Luo, A. Auggustsson, S. Kashtanov, J.-E. Rubensson, D. K. Shuh, H. Agren, and J. Nordgren, Phys. Rev. Lett. 91, 157401 (2003).

${ }^{13}$ L. Zoranić, F. Sokolić, and A. Perera, J. Chem. Phys. 127, 024502 (2007).

${ }^{14}$ J. G. Kirkwood and F. Buff, J. Chem. Phys. 19, 774 (1950).

${ }^{15}$ A. Ben-Naim, J. Chem. Phys. 67, 4884 (1977).

${ }^{16} \mathrm{~A}$. Ben-Naim, Statistical Thermodynamics for Chemists and Biochemists (Plenum, New York, 1992).

${ }^{17}$ E. Matteoli and G. A. Mansoori, Fluctuation Theory of Mixtures (Taylor \& Francis, New York, 1990).

${ }^{18}$ Y. Marcus, Monatsh. Chem. 132, 1387 (2001).

${ }^{19}$ E. Ruckenstein and I. Shulgin, Fluid Phase Equilib. 180, 281 (2001).

${ }^{20}$ I. L. Shulgin and E. Ruckenstein, J. Chem. Phys. 123, 054909 (2005).

${ }^{21}$ P. E. Smith, Biophys. J. 91, 849 (2006).

${ }^{22}$ J. P. O'Connell, Mol. Phys. 20, 27 (1971).

${ }^{23}$ A. Ben-Naim, A. M. Navarro, and J. M. Leal, Phys. Chem. Chem. Phys. 10, 2451 (2008).

${ }^{24}$ E. Matteoli and L. Lepori, J. Chem. Phys. 80, 2856 (1984).

${ }^{25}$ A. Perera and R. Mazighi, J. Chem. Phys. 143, 154502 (2015).

${ }^{26}$ R. Privat and J.-N. Jaubert, Chem. Eng. Sci. 82, 319 (2012).

${ }^{27}$ E. Matteoli, J. Phys. Chem. B 101, 9800 (1997).

${ }^{28}$ I. L. Shulgin and E. Ruckenstein, J. Phys. Chem. B 110, 12707 (2006).

${ }^{29}$ E. A. Ploetz, N. Bentenitis, and P. E. Smith, J. Chem. Phys. 132, 164501 (2010).

${ }^{30}$ P. M. Chaikin and T. C. Lubensky, Principles of Condensed Matter Physics (Cambridge University Press, 1995).

${ }^{31}$ L. Berthier and M. D. Ediger, Phys. Today 69(1), 40 (2016).

${ }^{32}$ W. L. Jorgensen, J. M. Briggs, and M. L. Contreras, J. Phys. Chem. 94, 1683 (1990).

${ }^{33}$ B. Chen, J. J. Potoff, and J. I. Siepman, J. Phys. Chem. B 105, 3093-3104 (2001).

${ }^{34}$ M. Mijaković, K. D. Polok, B. Kežić, F. Sokolić, A. Perera, and L. Zoranić, Mol. Simul. 41, 9 (2015).

${ }^{35}$ D. van der Spoel, E. Lindahl, B. Hess, G. Groenhof, A. E. Mark, and H. J. C. Berendsen, J. Comput. Chem. 26, 1701 (2005).

${ }^{36}$ J. M. Martínez and L. Martínez, J. Comput. Chem. 24(7), 819-825 (2003); L. Martínez, R. Andrade, E. G. Birgin, and J. M. Martínez, ibid. 30(13), 2157-2164 (2009).

${ }^{37}$ J. P. Hansen and I. R. McDonald, Theory of Simple Liquids (Academic, London, 1986).

${ }^{38}$ E. Oran Brigham, The Fast Fourier Transform (Prentice Hall, 1988).

${ }^{39}$ J. L. Lebowitz and J. K. Percus, Phys. Rev. 122, 1675 (1961).

${ }^{40}$ A. Perera and F. Sokolić, J. Chem. Phys. 121(22), 11272 (2004).

${ }^{41}$ B. Kežić and A. Perera, J. Chem. Phys. 137, 014501 (2012).

${ }^{42}$ F. H. Stillinger, J. Chem. Phys. 38, 1486 (1963).

${ }^{43}$ G. C. Benson and H. D. Pflug, J. Chem. Eng. Data 15, 382-386 (1970).

${ }^{44}$ J. C. Pings and J. Waser, J. Chem. Phys. 48, 3016 (1968).

${ }^{45}$ International Tables for Crystallography, edited by E. Prince (International Union of Crystallography, 2006), Vol. C.

${ }^{46}$ C. Andreani, M. Nardone, F. P. Ricci, and A. K. Soper, Phys. Rev. A 46, 4709 (1992).

${ }^{47}$ J. J. Weis and D. Levesque, Phys. Rev. A 13, 450 (1976).

${ }^{48}$ M. Tomšić, A. Jamnik, G. Fritz-Popovski, O. Glatter, and L. Vlcek, J. Phys. Chem. B 111, 1738 (2007).

${ }^{49}$ C. Bores, E. Lomba, A. Perera, and N. Almarza, J. Chem. Phys. 143, 084501 (2015).

${ }^{50} \mathrm{R}$. Lumry and S. Rajendar, Biopolymers 9, 1125 (1970).

${ }^{51}$ R. R. Krug, W. G. Hunter, and R. A. Grieger, Nature (London) 261, 566 (1976).

${ }^{52}$ H. Qiang and J. J. Hopfield, J. Chem. Phys. 105, 9292 (1996).

${ }^{53}$ K. Sharp, Protein Sci. 10, 661 (2001).

${ }^{54}$ R. Lumry and R. B. Gregory, in The Fluctuating Enzyme, edited by G. R. Welch (Wiley, New York, 1986), pp. 1-190.

${ }^{55}$ A. Perera, A. Rispe, L. Zoranić, R. Mazighi, and F. Sokolić, Mol. Phys. 107, 1349 (2009).

${ }^{56}$ A. Perera, Mol. Phys. 107, 487 (2009).

${ }^{57}$ B. Kežić, S. Dartois, and A. Perera, Mol. Phys. 113, 1108 (2015).

${ }^{58}$ A. Stradner, H. Sedgwick, F. Cardinaux, W. C. K. Poon, S. U. Egelhahf, and P. Shurtenberger, Nature (London) 432, 492 (2004). 
${ }^{59}$ G. Malescio and G. Pellicane, Nat. Mater. 2, 97 (2003).

${ }^{60}$ B. Kežić and A. Perera, J. Chem. Phys. 137, 134502 (2012).

${ }^{61}$ L. Zoranić, R. Mazighi, F. Sokolić, and A. Perera, J. Phys. Chem. 111, 15586 (2007).

${ }^{62}$ A. Perera, R. Mazighi, and B. Kežić, J. Chem. Phys. 136, 174516 (2012).

${ }^{63}$ C. S. Santos, H. V. R. Annapureddy, N. S. Murthy, H. K. Kashyap, E. W. Castner, and C. J. Margulis, J. Chem. Phys. 134, 064501 (2011).
${ }^{64}$ A. Triolo, O. Russina, B. Fazio, R. Triolo, and E. Di Cola, Chem. Phys. Lett. 457, 362 (2008).

${ }^{65}$ H. J. Jiang, P. A. FitzGerald, A. Dolan, R. Atkin, and G. G. Warr, J. Phys. Chem. B 118, 9983 (2014).

${ }^{66}$ G. D'Arrigo, R. Giodano, and J. Teixeira, Eur. Phys. J. E 10, 135 (2003).

${ }^{67}$ G. D'Arrigo, R. Giodano, and J. Teixeira, Eur. Phys. J. E 29, 37 (2009).

${ }^{68}$ M. Gruebele and D. Thirumaial, J. Chem. Phys. 139, 121701 (2013).

${ }^{69}$ H. Oshima and M. Kinoshita, J. Chem. Phys. 142, 145103 (2015). 